\title{
Solar Divergence Collimators for Optical Characterisation of Solar Components
}

\author{
D. Fontani, P. Sansoni, E. Sani, S. Coraggia, D. Jafrancesco, and L. Mercatelli \\ Consiglio Nazionale delle Ricerche, National Institute of Optics INO, Largo E. Fermi 6, 50125 Firenze, Italy \\ Correspondence should be addressed to P. Sansoni; paola.sansoni@ino.it
}

Received 14 November 2012; Accepted 21 December 2012

Academic Editor: Ho Chang

Copyright (C) 2013 D. Fontani et al. This is an open access article distributed under the Creative Commons Attribution License, which permits unrestricted use, distribution, and reproduction in any medium, provided the original work is properly cited.

\begin{abstract}
Experimentation and laboratory optical tests on solar components are central aspects of the research on renewable energies. The key element of the proposed testing systems is a solar divergence collimator, which exactly reproduces in laboratory the sunlight divergence, while commercial solar simulators are mainly aimed to replicate intensity and spectrum of the sun. Precise solar divergence reproduction is essential to correctly assess the optical properties and to simulate the operative conditions of a solar collecting device. Optical characterisation and experimentation can give information about production quality and homogeneity; moreover, specific tests can address the serial production of solar components detecting defects type and location. For Concentrating Photovoltaic systems, appropriate tests can analyze solar concentrators of various shapes, dimensions, and collection features. Typically, to characterise a solar component the most important and commonly examined quantities are collection efficiency, image plane analysis, and angle dependence.
\end{abstract}

\section{Introduction}

Solar energy concentration on reduced size surfaces, typically photovoltaic cells or more rarely optical fibres, represents a recent field of application within the renewable energy studies. These technologies and solar plants appear to be quite promising for energetic supply [1-4]. Optical systems for solar light concentration on reduced size surfaces were analyzed and experimented in our research laboratory since 1997 [5-9]. Our work on renewable energies includes three main research lines: optics to concentrate and transfer solar light by optical fibres, solar concentrators coupled to PhotoVoltaic cells (Concentrating PhotoVoltaic systems), and finally sunlight collectors coupled to various devices. These solar components can be applied in the photovoltaic field or for internal illumination.

Laboratory experimentation and optical measurements [5-7], on commercial solar components or samples realized from customized optical designs, represent crucial aspects of these researches. Optical characterization tests [10-12] can give information about production quality and realisation homogeneity of solar devices constituents. Dedicated and adapted measurements can address the serial production of solar components individuating type and location of realisation imperfections. For Concentrating PhotoVoltaic applications, suitable optical measurements can control the homogeneity of collector production, comparing the optical features among the different samples. Finally, to check the fidelity of reproduction of the original design, the optical characterization can be used to compare the measured values of optical properties to the nominal values belonging to the optical project of the component.

Solar simulators are often employed to optically characterise solar components: in fact they are more frequently used to test absorbers than collectors. However, due to the recent technological improvements in Concentrating Photovoltaics (CPV systems), there is an increasing request of employing solar simulators to test CPV components.

Commercial solar simulators [13-15] are essentially designed for experimentations on plane solar panels or single 


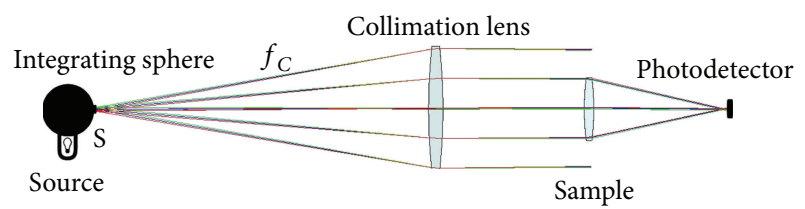

FIGURE 1: Scheme of collimator S1 (for image tests and focused image meas. on small components).

cells, without collection optical system. In principle, a solar simulator is a device that can reproduce, on limited areas, the conditions of solar irradiation with specific spectral characteristics and irradiance uniformity. The solar simulators on the market perform a quite good reproduction of spectral distribution and intensity of the solar emission: up to 200x for continuous light and up to 5000x for pulsed light. The main drawback of these solar simulators is that the output light has a divergence larger than the solar one: semi-angle divergence of few degrees, while the solar value is around $0.25^{\circ}$. The most sophisticated, so very expensive, device present on the market can produce a collimated beam with divergence halfangle $2.4^{\circ}$ and diameter $6^{\prime \prime}$ or divergence half-angle $1.2^{\circ}$ and diameter $12^{\prime \prime}$.

Image light distribution is another crucial aspect that must be taken into account, especially for the experimentation on concentrating photovoltaic components. In order to minimise the thermal stresses and to maximise the conversion efficiency, the CPV cells require adequate irradiance uniformity over the cell surface.

This paper proposes a solar divergence collimator able to exactly reproduce the solar divergence and to provide an extremely uniform beam. Our solar divergence collimator is applied to test solar components in a laboratory setup, whose main advantages are flexibility and versatility. A possible engineering of our test system, to obtain a closed and fixed instrument, would lose its ability to adapt the tests to specific requirements of the solar application or particular characteristics of the examined sample.

\section{Solar Divergence Collimators}

The optical collimator reproducing the solar divergence represents the core of every laboratory set-up employed to characterize solar components. The principal layouts for our optical tests are presented in Figures 1, 2, 3, and 4. The source is always realized by a white light illuminator coupled by an optical fibre to an integrating sphere. The solar divergence is accurately reproduced combining the sphere output diameter $S$ to the focal distance $f_{C}$ of the collimation optics, which can be a lens or a mirror.

The solar divergence collimator can be realized in axis (S1-S2) if the examined collectors have reduced dimensions. Collimator S1-S2 is presented in Figures 1 and 2, showing the two optical configurations used for image tests and collection efficiency assessment. The setup of S1-S2 includes a collimation lens with focal length $f_{C}=700 \mathrm{~mm}$ and diameter $d_{C}=90 \mathrm{~mm}$.

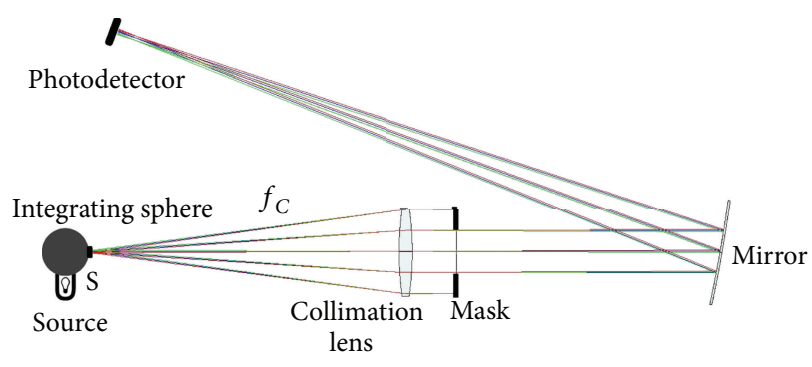

FIGURE 2: Scheme of collimator $S 2$ (for entering light measurement on small components).

For practical reasons, the test of solar collectors with diameter exceeding $80 \mathrm{~mm}$ is realized employing the solar divergence collimator L1-L2 in Figures 3 and 4. To clarify the schemes, in Figures 3 and 4 the values of $f_{C}$ and $d_{C}$ do not respect the real proportions, whereas they are correctly proportioned in Figures 1 and 2. Collimator L1L2 has two spherical mirrors with $f_{C}=1500 \mathrm{~mm}$ and $d_{C}=250 \mathrm{~mm}$. The first acts as collimation mirror while the second is a concentrating mirror (indicated as "Mirror" in $S 2$ Figure 2 and L2 Figure 4). In collimator L1-L2 the optical axis is deflected once (L1 Figure 3) or twice (L2 Figure 4) over the measurement plane. The bent configuration of L1L2 introduces some optical errors and aberrations on the collimated beam.

The verification of the solar divergence reproduction can be preliminary performed comparing the obtained beam diameter with the expected value. In practice we realise a reference target, a circle with the exact beam dimensions at the distance of the concentrating mirror ("Mirror" in Figures 4 and 2). The reference target is placed at this distance on the beam axis. The set-up L2 (or S2) projects a beam on it, which should correspond to the target circle. A more precise control of beam properties and solar divergence reproduction is represented by the beam measurements described in Section 4.

Concerning the optical quality of the beams, it is important to remark that the optical layout of S1-S2 is bent once in Figure 2, but the measured quantity is the entering light, which is filtered (by the mask) in the axial collimator. Then the mirror, bending the optical axis, concentrates the light over the photodetector. In collimator L1-L2 the collimation mirror deflects the optical axis realizing a bent collimator with two axes forming an angle $\alpha$. Then the concentrating mirror focuses the light on the sensor, introducing a second deflection of the optical axis (Figure 4). The crucial bend is the first one since it affects the quality of the collimated beam with solar divergence. Moreover the use of an integration sphere and the precision in realizing its aperture $S$ represent two other sources of optical errors.

\section{Aberrations of Collimated Beams}

The collimators under study are of two types: S1-S2 contains a collimation lens, while $L 1-L 2$ has a collimation mirror in a bent configuration. Both collimators generate a series of 


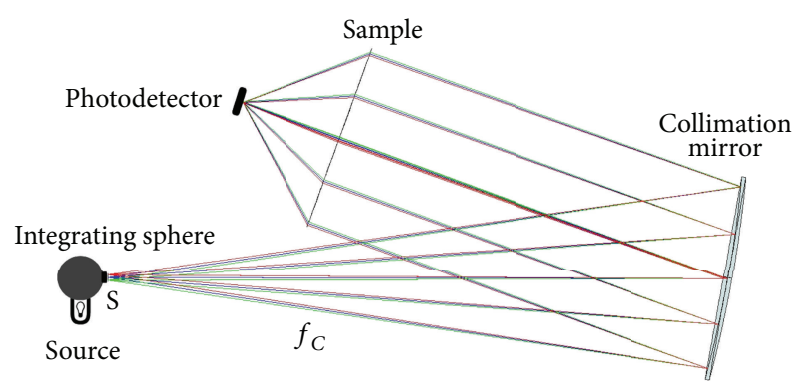

FIgURE 3: Scheme of set-up L1 (for image tests and focused image meas. on large components).

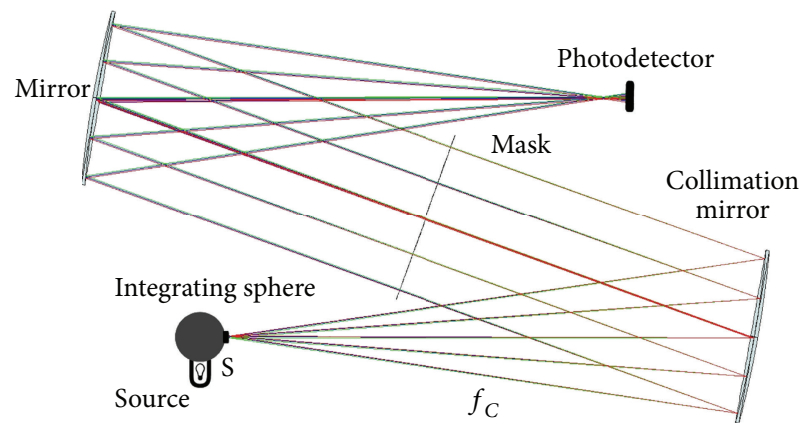

FIGURE 4: Scheme of set-up L2 (for entering light measurement on large components).

aberrations that contribute to degrade the definition of the image on the detector. The value of these aberrations must be quantified in order to identify the specific contribution generated by the sample under test. The use of collimator S1S2 allows limiting the aberrations by an appropriate choice for the collimation lens, which should be a lens with field correction and optimized for the used spectral bandwidth. On the other hand, employing collimator L1-L2 the aberrations mainly depend on the collimator angle $\alpha$ and on the surface type of the collimation mirror. In L1-L2 of Figures 3 and 4 the collimation mirror was chosen to be spherical because it is easy and cheap to be realized. The aberration study was carried out considering a spherical mirror of focal length $1500 \mathrm{~mm}$ and an ideal lens with focal length $250 \mathrm{~mm}$, which represents the component under test. The ideal lens allows analyzing the aberrations of the wave front coming out from the collimation mirror in the position where the solar collectors are usually tested. The aberrations of the beam generated by $L 1-L 2$ are reported in Figure 5 as standard Zernike coefficients $C_{Z}$, considering three collimator angles $\alpha: 5^{\circ}, 15^{\circ}$, and $25^{\circ}$. The actual angle of the collimator L1L2 used in our laboratory for collector tests is $15^{\circ}$ and the laboratory set-up allows angles up to $23^{\circ}$. The values of spherical aberration, coma, astigmatism, and field curvature are plotted versus the entrance pupil diameter of the test lens (in millimetres), which corresponds to the beam diameter under examination.

The $C_{Z}$ values plotted in Figure 5 indicate that the main contribution is represented by the astigmatism and it improves with the diameter of the collimated beam. For beam diameter lower than $150 \mathrm{~mm}$ and $\alpha \leq 15^{\circ}$ the $C_{Z}$ coefficients are such that the effect of the single aberrations on the quality of the image produced by the lens is negligible. The image maintains high uniformity in intensity.

The degradation of image definition was estimated simulating a collimator $L 1-L 2$ with a ray-tracing optical design software, which provided simulations reproducing the profile of the image Sg of our source. The profiles of the image Sg are displayed in Figures 6(a) and 6(b) for angles $\alpha$ up to $25^{\circ}$ and for two beam diameters. In Figure 6(a) the beam diameter is $150 \mathrm{~mm}$, while Figure 6(b) refers to a beam diameter of $250 \mathrm{~mm}$ that corresponds to the total width of the solar divergence beam. For $\alpha \leq 15^{\circ}$ the image keeps the rectangular shape: hence it can be concluded that our collimator L1-L2 is a very good test system for sample diameters up to $150 \mathrm{~mm}$ with $\alpha=15^{\circ}$.

As final validation, the reliability of the off-axis collimator L1-L2 to test solar collectors was experimentally verified. This check is reported in Figure 7 comparing the characteristics of the image produced by an ideal lens, without aberrations, with the image generated by a real solar collector. The comparison of these two optical elements is useful to evaluate the contribution of the aberrations characterizing the offaxis spherical mirror in order to separate them from the contributions due to the tested collector.

Figure 7 compares the profile of a real image to the profile of a simulated image. The measured image was acquired by a CCD camera with a Fresnel lens of diameter $180 \mathrm{~mm}$ and focal length $195 \mathrm{~mm}$. The simulated curve in Figure 7 is obtained for a lens with the same optical features, but considered an ideal lens without aberrations. For the real lens the aberrations contribution is visible on the image profile, which results enlarged at the bottom and more smoothed and restricted at the top of the curve. The shape of the ideal lens image is almost rectangular, while the measured image profile approaches a bell shape. It can thus be concluded that for the examined measurements the aberrations of collimator L1L2 can be considered negligible. It is necessary to repeat this comparison operation at every new collector test in order to verify the degree of interaction of the measurement system with the parameters of the sample to be measured.

\section{Measurements of Collimated Beams}

The research is completed by a set of experimental measurements examining the solar divergence beam generated by collimators S1-S2 and L1-L2. The detection is preformed sampling the beam by a photodiode (of side $4 \mathrm{~mm}$ ) displaced on the vertical plane XY. The sensor is mounted on a motorized translation stage, which is shifted by another translation stage that is perpendicularly mounted. Suitable sampling step and speed were chosen to reach a good tradeoff between high definition of acquired image and acceptable length of measurement time. The beam of collimator S1-S2 has diameter $90 \mathrm{~mm}$; hence it can be completely sampled by our detection system, which has a maximum excursion of $150 \mathrm{~mm}$. For collimator L1-L2 the beam has diameter $250 \mathrm{~mm}$, 


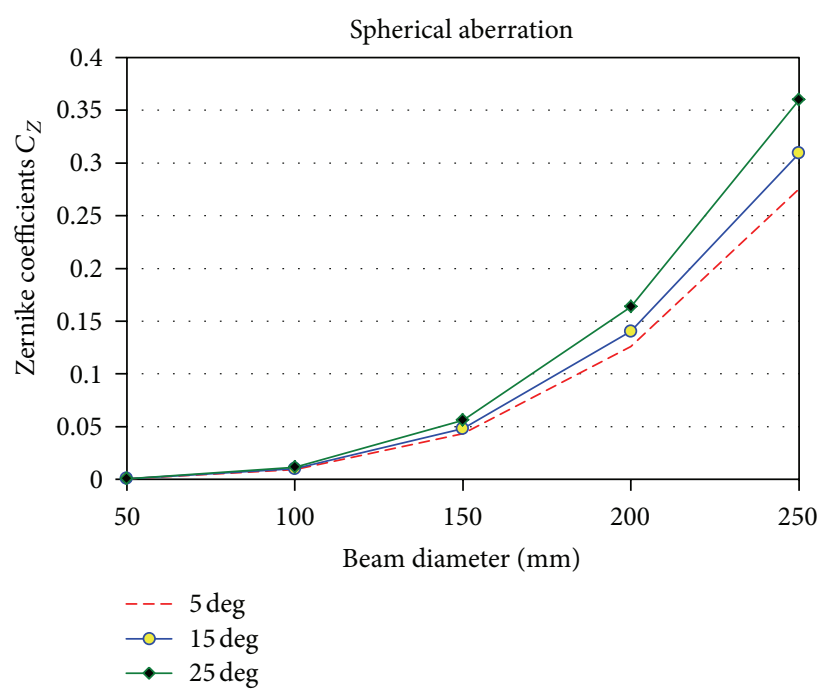

(a)

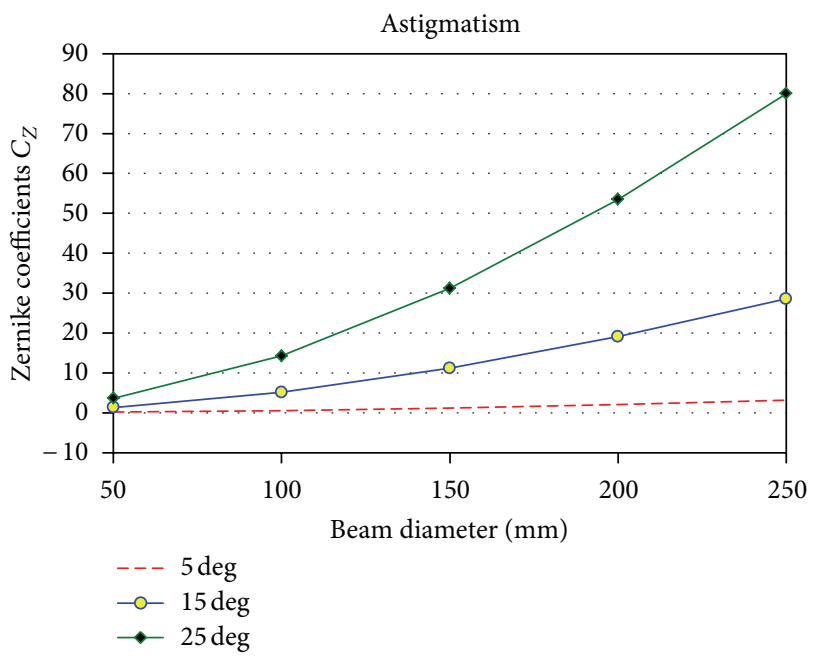

(c)

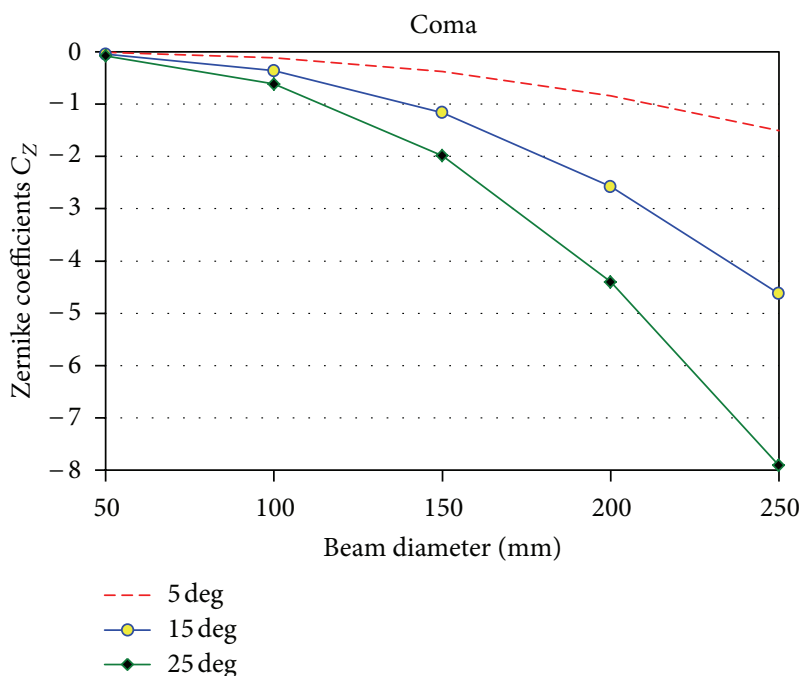

(b)

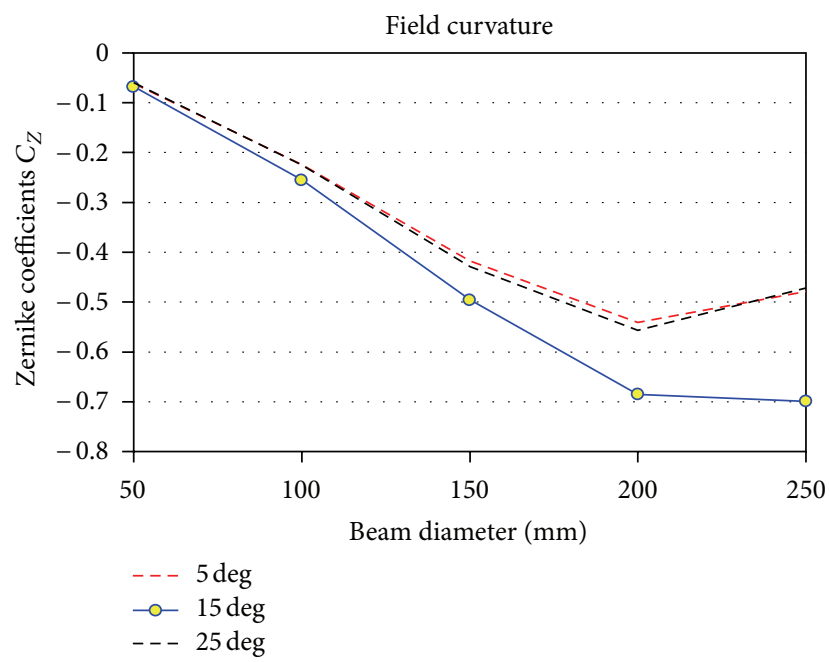

(d)

Figure 5: Aberrations as Zernike coefficients for $\alpha=5^{\circ}-25^{\circ}$.

therefore the measurement procedure is more complicated. Two photodiodes are mounted, at a horizontal distance of $150 \mathrm{~mm}$, on the same translation stage and they are displaced together. In this way two parallel sampling are obtained in correspondence of the left and right portion of the beam. To take into account possible differences between the two sensors, the double sampling is repeated exchanging the photodiodes. The final image is then calculated averaging the results of the two measurements.

Figure 8 shows the solar divergence beam measured on collimator S1-S2: the left picture presents a 3D plot, while the right picture reports a $2 \mathrm{D}$ image of the beam. Analogously Figure 9 presents the 2D and 3D plots of the measurements on the beam of collimator L1-L2.

In the measurements on the collimated beam of $L 1-L 2$ the vertical sampling range is $150 \mathrm{~mm}$ while the beam diameter is $250 \mathrm{~mm}$, so the images represent only the central portion of the beam. Nevertheless the sampling is sufficient to contain the beam in the horizontal direction and the aberrations affect this central band of the solar divergence beam.

By a practical point of view, in our laboratory set-up L1L2 the minimum collimator angle $\alpha$ is $15^{\circ}$. This is the value usually employed for the solar component tests, because it minimizes the aberration effects. The beam in Figure 9 does not show aberration effects, which result to be lower than the detection sensitivity.

It can be concluded that both collimators are appropriate to test solar components: the optical quality of the solar divergence beams is very high for S1-S2 and high for L1L2. For collimator S1-S2 inside the diameter of $90 \mathrm{~mm}$ the beam maintains very high uniformity in intensity with profile variations within $10 \%$ of the maximum value. For the beam of collimator $L 1-L 2$, the data fluctuations are lower than $10 \%$ in the diameter $180 \mathrm{~mm}$ and lower than $20 \%$ in the diameter $210 \mathrm{~mm}$. Consequently the maximum useful diameter for accurate measurements on the beam obtained with $L 1-L 2$ can 


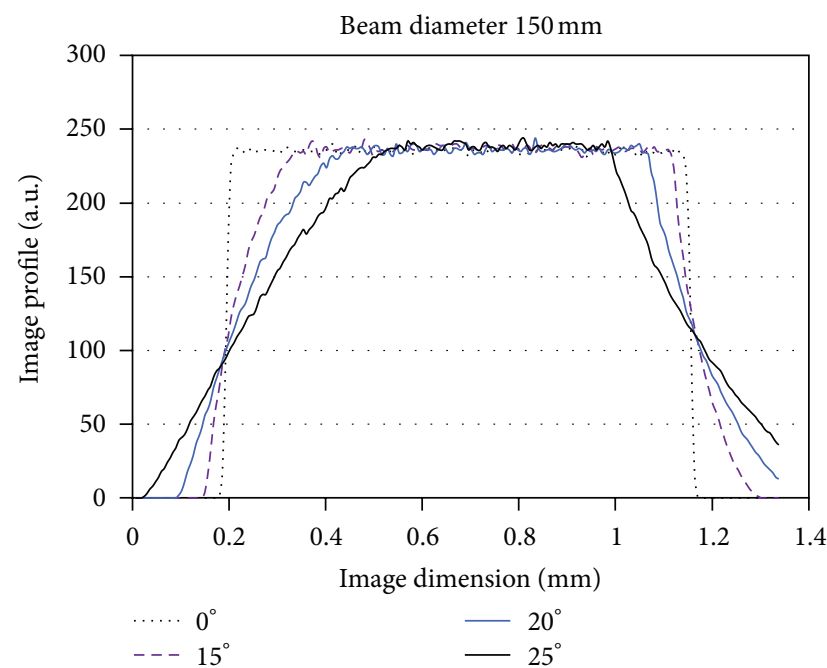

(a)

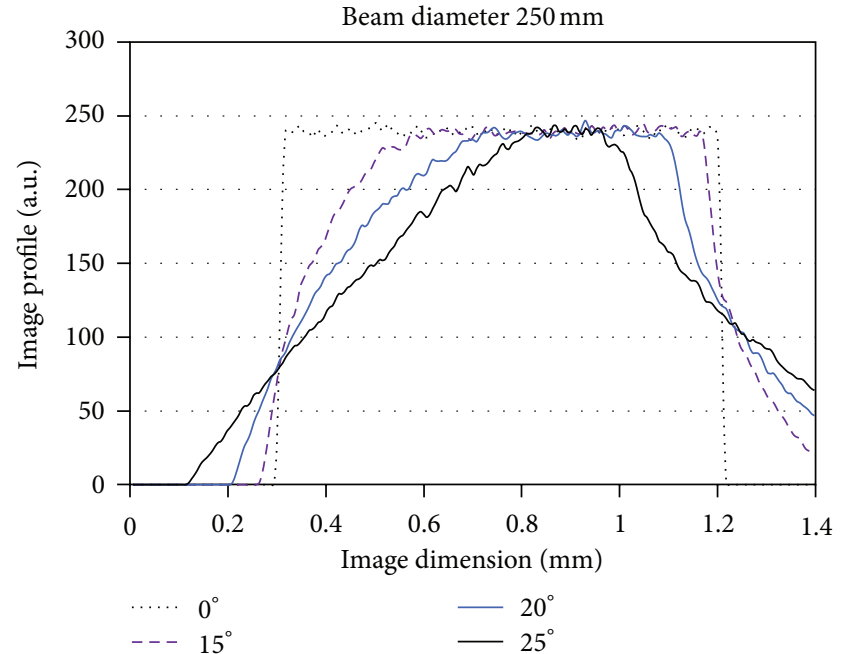

(b)

FIGURE 6: Image profiles for beam diameter $150 \mathrm{~mm}$ (a) or $250 \mathrm{~mm}$ (b).

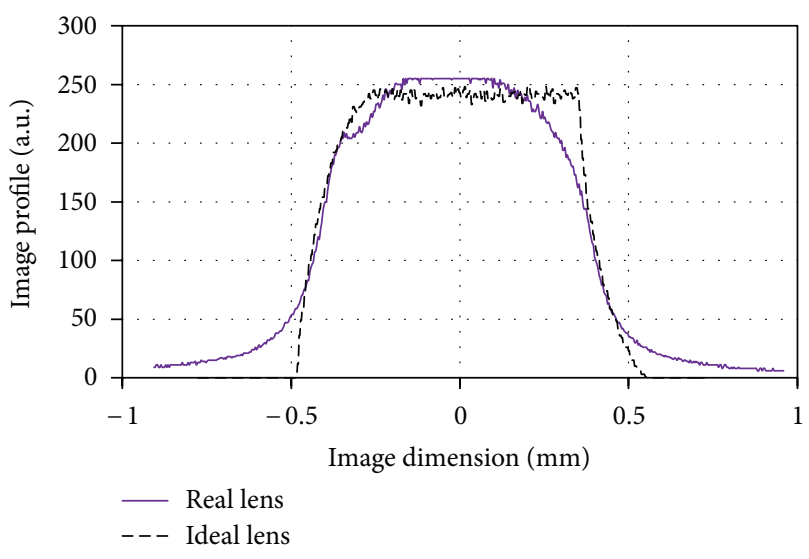

Figure 7: Profiles of simulated and detected images.

be considered $210 \mathrm{~mm}$, while high accuracy measurements should be performed within the diameter of $180 \mathrm{~mm}$.

Two aspects could be improved in the described optical systems that reproduce the solar divergence. The first aspect concerns the precise value chosen for the beam divergence: the solar divergence, as total angular aperture, ranges from $0.5253^{\circ}$ (Aphelion) to $0.5421^{\circ}$ (Perihelion). The second aspect is the reproduction of the solar spectrum, typically performed using halogen or Xenon lamps. Figure 10 presents the spectra of the light emitted by several sources, comparing the measurements to the sunlight spectrum. All measurements are performed coupling the source to the integrating sphere. The examined lamps cover the wavelength range of the solar spectrum (300 nm-1500 nm), but with a different spectral distribution. Moreover in laboratory we use an optical fibre that selects almost the visible light $(350 \mathrm{~nm}-800 \mathrm{~nm})$, which is typically the range of use and test of solar components. The internal source of the two illuminators is a halogen lamp with an elliptic mirror: its spectrum corresponds to the illuminator without optical fibre (in Figure 10).

In our laboratories the solar divergence beams of Figures 8 and 9 are successfully applied as testing light to characterize solar components. In particular we developed test configurations and procedures to test solar collectors with maximum diameter/diagonal $240 \mathrm{~mm}$. For such reduced sizes the examined samples typically have various shapes, dimensions, and collection characteristics. Therefore each solar image presents different light levels and uniformity features, which require the use of appropriate instrumentation and measurements procedures.

The described optical measurements on solar collectors consider image plane analysis, angle dependence, collection efficiency, and spot size. The proposed characterization provides optical properties and collection performances, which are crucial to optimise a solar exploitation device. However on the base of the specific solar application it can be useful to examine other aspects or assess specific quantities significant for the examined case [16-21].

\section{Image Plane Analysis}

Considering the characterisation of a CPV solar collector, some preliminary studies on the focal distance $f$ are essential both for image analyses and for collection efficiency assessment. It is essential to assess the position of each focal plane, measuring the experimental values of $f$ for each collector sample. The focal plane definition is dictated by collector design and solar system application. Solar collectors are optically designed as nonimaging systems with the main purpose of maximizing the focused energy within the nominal image. Therefore the focal plane can simply be individuated by maximizing the collected light over the whole examined plane (for absorber size exceeding the nominal 


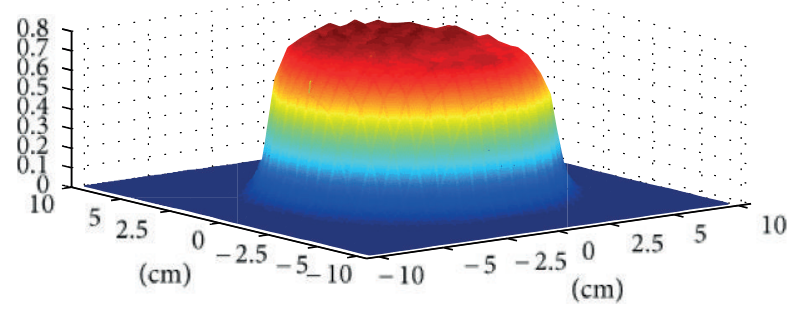

(a)

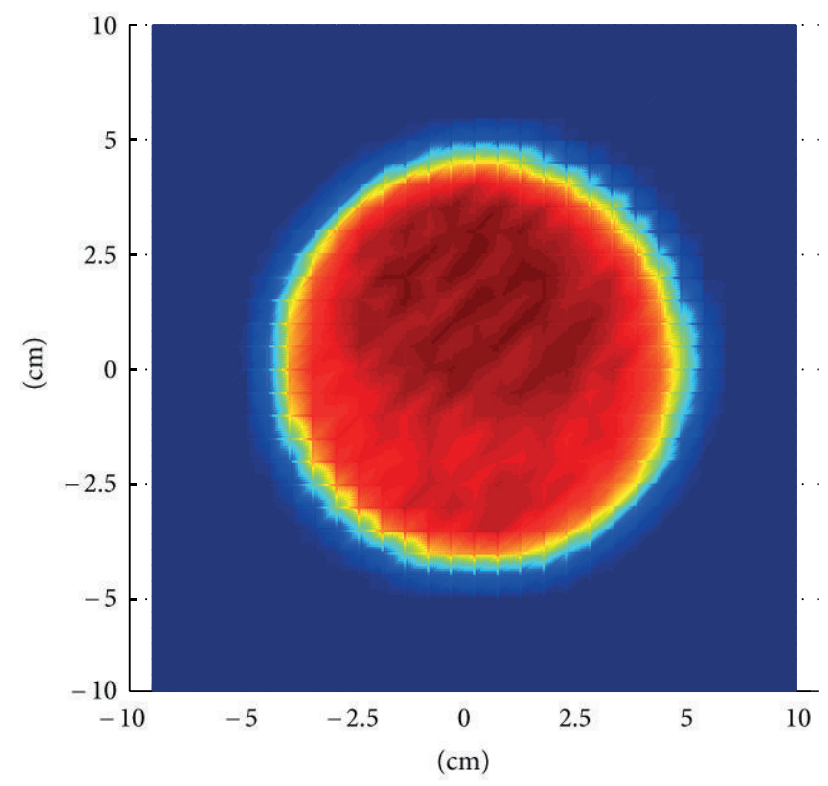

(b)

Figure 8: Measurement of the S1-S2 beam: 3D and 2D plots.

image). If the dimension of the absorber is crucial, as in case of photovoltaic cells (or optical fibres), the image plane can be defined maximizing the light concentrated over a detector with shape and size of the real absorber. After the determination of the focal distance, several experimental measurements can then be performed to examine the image generated by each sample of a solar collector production. In particular for Concentrating PhotoVoltaic applications it is fundamental to analyze the light distribution in the image plane.

The key element of our optical tests system is the solar divergence collimator reproducing the divergence of sunlight rays. It can be of two types: collimator S1-S2 (Figures 1 and 2) for small components, with diameter/diagonal lower than $80 \mathrm{~mm}$, or collimator L1-L2 (Figures 3 and 4) for larger samples, up to diameters of $240 \mathrm{~mm}$. Figures 1 and 3 show the two optical systems $S 1$ and $L 1$ used for the image plane analysis. The difference between the two layouts is the collimation optics: a lens with focal length $f_{C} 700 \mathrm{~mm}$ in $S 1$ Figure 1 or a spherical mirror with $f_{C} 1.5 \mathrm{~m}$ in L1 Figure 3. Figure 11 illustrates the laboratory practical realization of solar divergence collimator L1.

The source for L1-L2 and S2-S2 is realized by a white light illuminator coupled to an integrating sphere using an optical

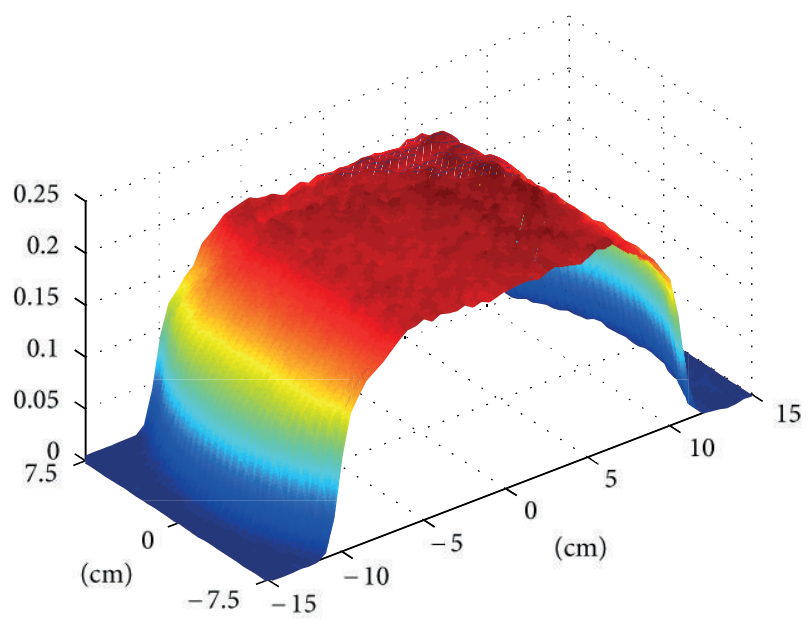

(a)

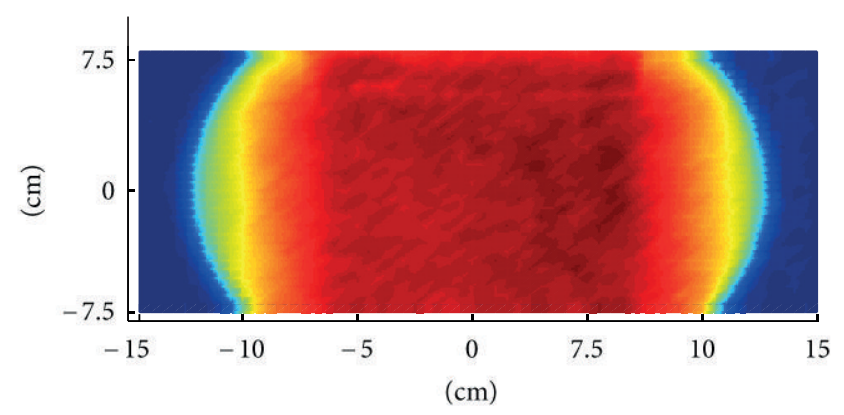

(b)

FIgURE 9: Measurement of the L1-L2 beam for $\alpha=15^{\circ}: 3 \mathrm{D}$ and 2D plots.

fibre. The solar divergence is simulated combining the sphere output diameter $S$ to the focal distance $f_{C}$ of the collimation optics. The beam with solar divergence impinges on the sample under test and a camera visualizes the generated image. The sensor used to estimate the focal distance and to examine the image plane is a CCD camera, while for the measurement of collection efficiency the sensor is a photodetector. Size and shape of the photodetector should correspond to those of the absorber.

The high-definition camera is mounted on a mechanical support that is usually displaced by a high-precision micrometric translation stage, to keep a good stability of the detection system and to allow a precise estimation of the focal distance. Beside the high precision, the translation stage should have high reproducibility of the positions and large excursion. In our laboratory optical system we use a CMOS camera with an external chip, which allows examining collectors with very short focal distances. Figure 12 shows the CMOS camera testing a component with a diameter of few centimetres. The image saturation is a critical parameter in these tests. First of all, the source level should be adapted to obtain a good image for the camera acquisition. The detection parameters can be properly chosen to evidence different aspects of the image analysis. For instance the image saturation must be avoided when determining the beam 


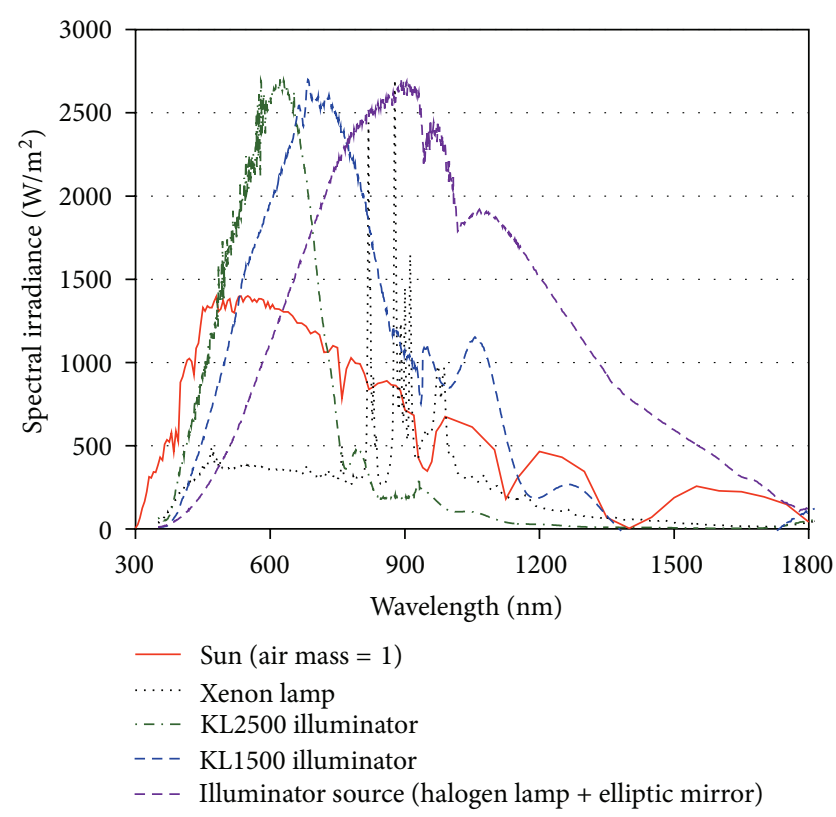

FIGURE 10: Lamp light (measured with integrating sphere) compared to the sunlight.

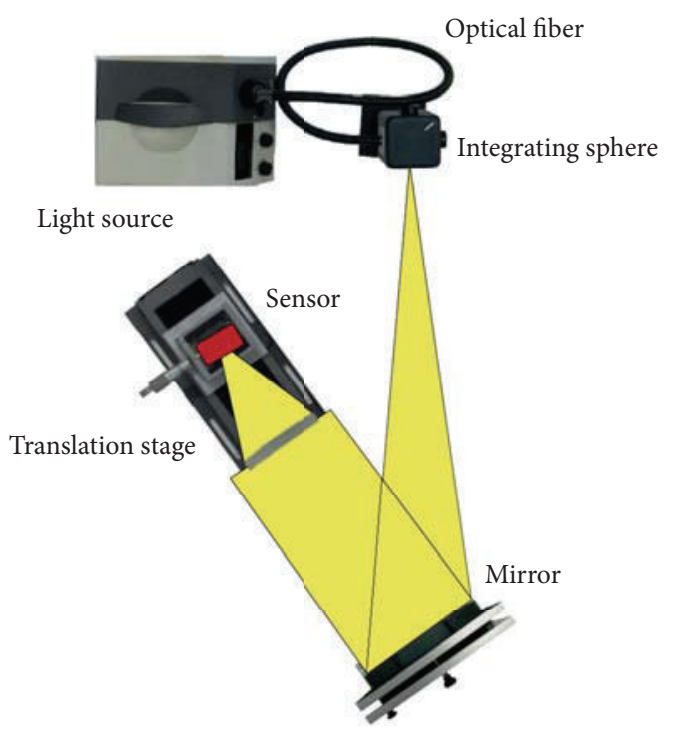

Figure 11: Practical realization of configuration L1 (for large components).

profile. On the other hand, the image saturation can be useful to emphasize image borders.

It is important to remark that all these measurements are very sensitive to misalignments between collector plane and camera array plane. Therefore mounting stability and alignment accuracy are essential elements to obtain reliable and reproducible results.

To consider some defocusing effects, the mentioned analysis can be repeated examining the parallel planes located before and after the focal position. Moreover dedicated

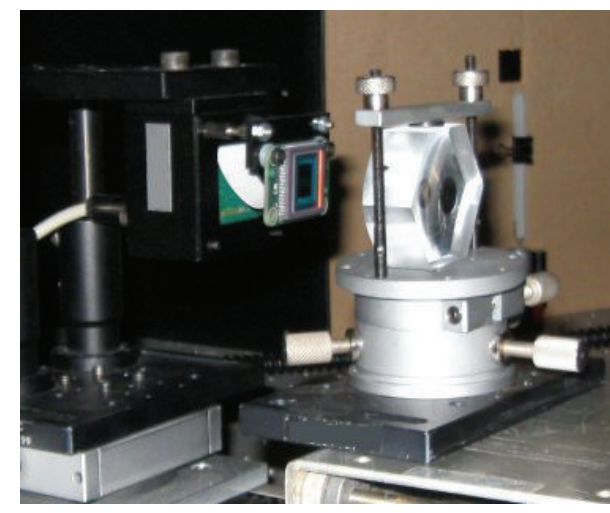

FIGURE 12: CMOS camera examining a small solar collector.

measurements can investigate the dependence on specific parameters of interest.

\section{Angle Dependence Study}

Suitably designed and especially realized configurations are dedicated to examine angular properties and the angular dependences of the tested components. These angular measurements assess how collector features and performances are affected by rotations, tilts, and angular misalignments in general. These crucial aspects are connected to collection efficiency performance, tracking errors and Field of View aperture of the optical system. In the worse case, the angular misalignment can cause bad illumination over the photovoltaic cell, generating thermal stresses.

Since every collector has its specific collection geometry, it is consequently characterized by specific angular effects and angle dependencies, which can be examined by appropriate tests. Furthermore typically each application has specific angular requirements that can be experimented or verified in laboratory by suitable tests on angle dependence.

Finally, these angular analyses can be combined with image plane studies or collection efficiency assessment, to complete the research and to provide a more realistic simulation. Investigating the interactions between the separately tested aspects, this experimentation represents a better simulation of the real working conditions.

The angular tests basically require a tilt or a rotation stage, which is combined to the instrumentation used for image plane analyses or collection efficiency tests.

By a practical point of view, for the simulation of mounting errors or angular misalignments only the collector is rotated, while in the tracking error tests both collector and detector are rotated with the same reference plane.

\section{Collection Efficiency Test}

For the applications of sunlight exploitation, the essential quantity to be considered is the collection efficiency. The measurements are performed on a white light collimator that reproduces the solar divergence assessing the efficiency of sunlight collection. The collection efficiency is obtained as 


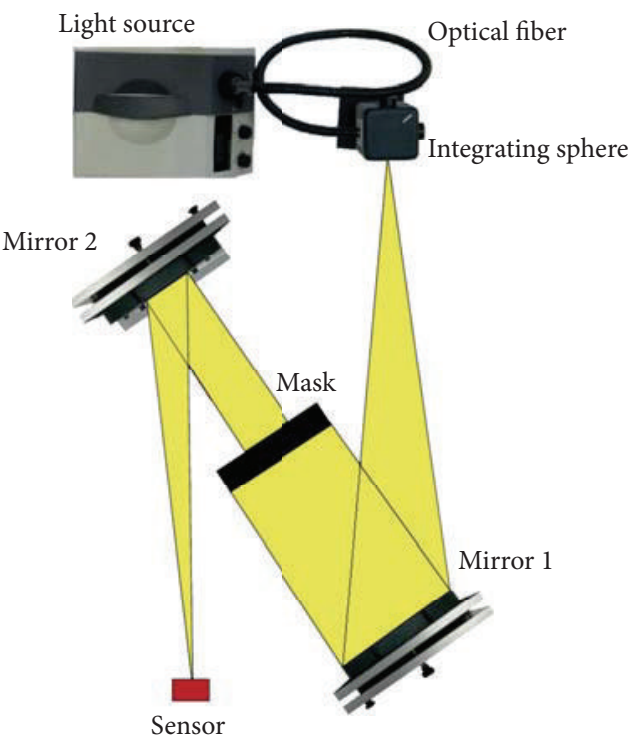

FIgURE 13: Practical realization of configuration L2 (for large components).

ratio between the light focused within the nominal image and the light inside the entrance aperture of the collector. These two quantities are separately measured. The focused light is measured using collimator $S 1$ of Figure 1 (for small collectors) or collimator L1 (for large collectors), shown schematically in Figure 3 and practically in Figure 11. For this measurement the sensor is a photodetector with dimensions corresponding to the nominal image, obtained from the optical project of the tested collector. The beam with solar divergence illuminates the sample under test, which is located at the focal distance to maximize the collected light.

The second measurement concerns the light entering through the collector entrance aperture. It is performed using collimator S2 in Figure 2 (for small collectors) or collimator L2 (for large collectors), reported as scheme in Figure 4 and as laboratory set-up in Figure 13. The beam with solar divergence impinges on a suitable mask, composed of a screen with a hole reproducing shape and size of collector entrance aperture. The light passing through the mask is concentrated by a spherical mirror and measured by a photodetector.

In general the optical tests are aimed to verify quality and homogeneity of collector production and reproducibility of the collector fabrication process. Every test checks production homogeneity and reproducibility for the specific aspect examined by the test; but probably the most significant results are represented by collection efficiency assessment and focal distance measurement.

The essential optoelectronic instrumentation employed to assess the collection efficiency is a photodiode with large dynamic amplifier, a mask (simulating the collector entrance aperture), a concentrating mirror (spherical mirror), and a translation stage. Optical systems S1 of Figure 1 and S2 Figure 2 are used to measure collectors with maximum diagonal/diameter $80 \mathrm{~mm}$. Components of larger dimensions are tested with configurations L1 of Figure 3 and L2 Figure 4.
The current mirrors available in our laboratory have diameter $250 \mathrm{~mm}$ and they allow testing samples with maximum diameter $240 \mathrm{~mm}$. The photodetector used in our laboratory set-up is a squared photodiode of side $18 \mathrm{~mm}$. In the measurement of entering light the image diameter of the source $S$ on the photodiode exceeds $15 \mathrm{~mm}$, therefore the photodiode should have suitable dimensions. This is due to the fact that the source diameter $S$ is dictated by the requirements of solar divergence reproduction and by the focal length of collimation optics.

An important aspect is to utilize the central portion of the collimated beam, characterized by higher uniformity. The reflectivity of the concentrating mirror should be assessed especially because they typically are large mirrors with diameters of $200-300 \mathrm{~mm}$. Moreover the reflectivity is not constant over the reflecting surface; hence we usually estimate an average value, which is successively employed to rescale the detection. Finally the entering light measurement can be replaced by a light density assessment if the beam is homogeneous enough.

\section{Image Size Measurement}

A simple and low-cost methodology allows evaluating the light concentrated into a fibre or over a photovoltaic cell. This experimental measurement assesses image spot dimension and light distribution within the focused image. The optical set-up for this image control includes a white light source reproducing the solar divergence. The beam impinges on the tested collector and a detection system is located in its image plane. It consists of a photodiode combined to a multi holes mask. Figure 14 shows an example of the multi hole mask with 8 hole diameters $d$, with approximate step of $0.1 \mathrm{~mm}$, suitable for images of few millimetres. The mask moves in front of the detector and it allows measuring the light intensity Id corresponding to the encircled image of the collector, which is the luminous intensity focused inside a hole. This image analysis can verify if the collector production is homogeneous for what concerns the light distribution inside the image. The results are expressed, in percentage, as ratio between the encircled image light and the total light in the focal plane. Some measurements results appear particularly interesting: the diameter corresponding to the nominal image of the collector and the value corresponding to the absorber (core diameter of the optical fibre or photovoltaic cell area).

The technique to determine the image diameter $\mathrm{Di}$ is based on the measurement of the encircled image light Id. The value of Di is obtained when the Id values start to saturate, thus indicating that the hole diameter $d$ is exceeding Di. Some Id results measured using a multi-hole mask are reported in Figure 15: the Id values are plotted versus the hole diameter $d$. For verification and comparison, Figure 15 also reports the corresponding values estimated from an image acquired by a CMOS camera. Considering a saturation threshold of 95\%, for the curve in Figure 15 the value of image diameter Di is $1.6 \mathrm{~mm}$.

In this test the translation stage moves the multi-holes mask behind the collector, which maintains its position. In 


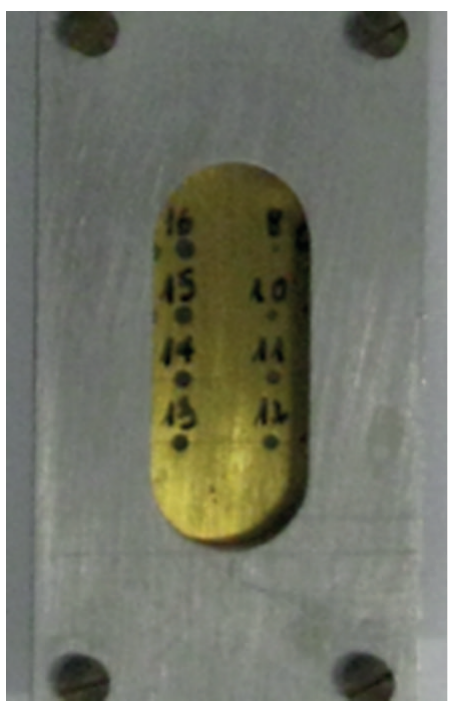

FIGURE 14: Example of Multi hole mask.

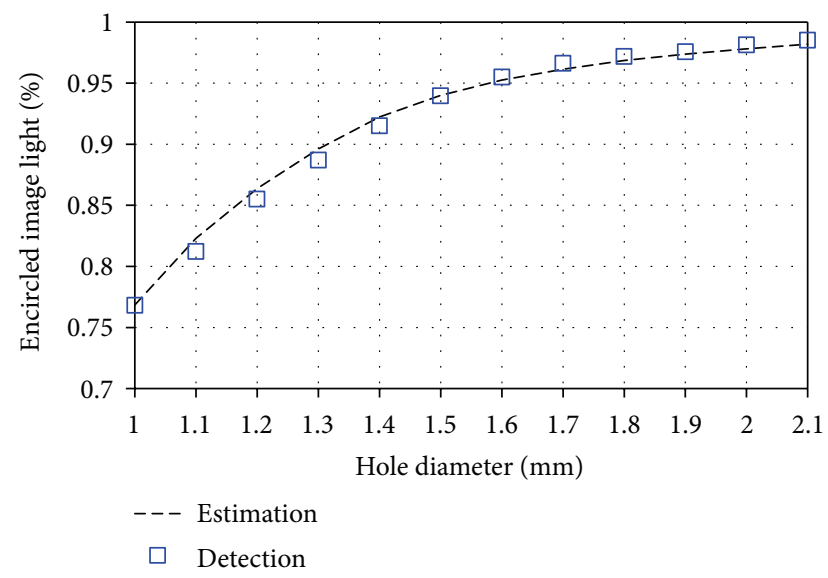

Figure 15: Measurement and calculation of the encircled image light.

fact, the most critical point for this measurement is the alignment of each hole. The multi-hole mask should be moved in the image plane, keeping the hole in the focal position, which is practically obtained maximizing the photodiode output signal.

This procedure can be applied when the image is unusable for the camera: in case of very luminous or extremely poor signal.

\section{Conclusions}

Since 1997 within our researches on renewable energies we studied and experimented solar concentrators for small surfaces. Our laboratory developed fibre-coupled collectors, Concentration PhotoVoltaic (CPV) systems, and optical systems coupled to various devices. These solar components were designed to be applied in the photovoltaic sector or for internal lighting.

For simulating the real working conditions of a direct exposition to the sun, collimators with solar divergence are employed in the optical systems to test solar components. The solar divergence is reproduced combining the output diameter of an integrating sphere to the focal distance of a collimation lens. The solar divergence collimator can be realized in axis for small collectors. While collectors with diameter/diagonal $>80 \mathrm{~mm}$ are tested using two large spherical mirrors with focal length $1.5 \mathrm{~m}$. In this case the collimation mirror deflects the optical axis realizing a bent collimator with two axes forming an angle $\alpha$. This bent configuration introduces some optical errors on the collimated beam with solar divergence. In the bent collimator the beam aberrations depend on the angle $\alpha$. Measurements of the solar divergence beams have evidenced that both collimators are appropriate to test solar components. The collimated beams do not show aberration effects that are lower than the detection sensitivity. The optical quality of the solar divergence beam is very high for the unbent collimator and high for bent collimator. Besides high accuracy measurements can be performed in the central part of the beam of the bent collimator.

Optical characterization and experimentation are crucial aspects for the development of every sunlight exploitation device and in particular for CPV systems. Optical tests can verify quality and homogeneity of solar components production or compare the measured values of optical features to the nominal values estimated in the optical design.

The core of our optical testing systems is a solar divergence collimator, which produces a beam with solar divergence and elevated illumination uniformity. The examined collectors can have various shape, dimensions, and concentration features. Hence each image presents different light levels and concentration features, which require the use of appropriate hardware and procedures. The described optical tests allow determining image plane analysis, angle dependence, collection efficiency, and image dimensions. The mentioned features represent an exemplificative and basic optical characterization for sunlight exploitation; however each solar application requires adapting the tests to examine the significant quantities and aspects.

Our research group developed optical systems and methodologies to characterize solar components of reduced size (max diameter/diagonal $240 \mathrm{~mm}$ ). This dimensional limitation on the examinable samples is only due to the actual size of our laboratory; the sample size can be increased extending the optical system and using mirrors with larger diameter. In alternative, samples larger than $240 \mathrm{~mm}$ can be examined in portions, averaging and combining the results to obtain the optical characterisation of the component. Another important case is the test of high-concentration collectors: the solution can be either to use a reduced source emission or to choose suitable sensors and acquisition parameters. However the major advantage of using a laboratory setup is its adaptability to sample features and solar application needs.

\section{Acknowledgment}

The authors wish to thank Dr. D. Ferruzzi for her fundamental contribution to develop the ray-tracking simulations 
of solar divergence collimators in order to assess beam aberrations and beam profile errors.

\section{References}

[1] R. M. Swanson, "The promise of concentrators," Progress in Photovoltaics, vol. 8, pp. 93-111, 2000.

[2] W. T. Welford and R. Winston, High Collection Nonimaging, Optics Academic Press, San Diego, Calif, USA, 1989.

[3] US Department of Energy/Electric Power Research Institute (EPRI), "Renewable energy technology characterisations," Topical Report TR-109496, 1997.

[4] A. Luque, Solar Celle and Optics for Photovoltaic Concentration, The Adam Higler Series on Optics and Optoelectronics, Taylor \& Francis, New York, NY, USA, 1989.

[5] P. Sansoni, F. Francini, and D. Fontani, "Optical characterisation of solar concentrator," Optics and Lasers in Engineering, vol. 45, no. 3, pp. 351-359, 2007.

[6] P. Sansoni, F. Francini, D. Fontani, L. Mercatelli, and D. Jafrancesco, "Indoor illumination by solar light collectors," Lighting Research and Technology, vol. 40, no. 4, pp. 323-332, 2008.

[7] F. Francini, D. Fontani, D. Jafrancesco, L. Mercatelli, and P. Sansoni, "Optical control of sunlight concentrators," in High and Low Concentration for Solar Electric Applications, vol. 6339 of Proceeding of SPIE, San Diego, Calif, USA, September 2006.

[8] P. Sansoni, D. Fontani, F. Francini, D. Jafrancesco, and G. Longobardi, "Optical design and development of fibre coupled compact solar collectors," Lighting Research and Technology, vol. 39, no. 1, pp. 17-30, 2007.

[9] C. Ciamberlini, F. Francini, G. Longobardi, M. Piattelli, and P. Sansoni, "Solar system for exploitation of the whole collected energy," Optics and Lasers in Engineering, vol. 39, no. 2, pp. 233246, 2003.

[10] P. Maddalena, A. Parretta, A. Sarno, and P. Tortora, "Novel techniques for the optical characterization of photovoltaic materials and devices," Optics and Lasers in Engineering, vol. 39, no. 2, pp. 165-177, 2003.

[11] A. Parretta, P. Grillo, P. Maddalena, and P. Tortora, "Method for measurement of the directional/hemispherical reflectance of photovoltaic devices," Optics Communications, vol. 186, no. 1-3, pp. 1-14, 2000.

[12] P. Horta, M. J. Carvalho, M. C. Pereira, and W. Carbajal, "Longterm performance calculations based on steady-state efficiency test results: analysis of optical effects affecting beam, diffuse and reflected radiation," Solar Energy, vol. 82, no. 11, pp. 1076-1082, 2008.

[13] D. L. Fain, "Design considerations for precision solar simulation," Applied Optics, vol. 3, no. 12, pp. 1389-1396, 1964.

[14] I. Powell, "New concept for a system suitable for solar simulation," Applied Optics, vol. 19, no. 2, pp. 329-334, 1980.

[15] C. Domínguez, I. Antón, and G. Sala, "Concentrator photovoltaics solar simulator," in Frontiers in Optics, p. JThA1, Optical Society of America, Rochester, NY, USA, 2008.

[16] J. L. Balenzategui and F. Chenlo, "Measurement and analysis of angular response of bare and encapsulated silicon solar cells," Solar Energy Materials and Solar Cells, vol. 86, no. 1, pp. 53-83, 2005.

[17] D. C. Miller and S. R. Kurtz, "Durability of Fresnel lenses: a review specific to the concentrating photovoltaic application,"
Solar Energy Materials and Solar Cells, vol. 95, no. 8, pp. 20372068, 2011.

[18] H. Zhai, Y. J. Dai, J. Y. Wu, R. Z. Wang, and L. Y. Zhang, "Experimental investigation and analysis on a concentrating solar collector using linear Fresnel lens," Energy Conversion and Management, vol. 51, no. 1, pp. 48-55, 2010.

[19] D. Jing, H. Liu, X. Zhang, L. Zhao, and L. Guo, "Photocatalytic hydrogen production under direct solar light in a CPC based solar reactor: reactor design and preliminary results," Energy Conversion and Management, vol. 50, no. 12, pp. 2919-2926, 2009.

[20] W. T. Xie, Y. J. Dai, and R. Z. Wang, "Numerical and experimental analysis of a point focus solar collector using high concentration imaging PMMA Fresnel lens," Energy Conversion and Management, vol. 52, no. 6, pp. 2417-2426, 2011.

[21] B. Perers, "An improved dynamic solar collector test method for determination of non-linear optical and thermal characteristics with multiple regression," Solar Energy, vol. 59, no. 4-6, pp. 163$178,1997$. 

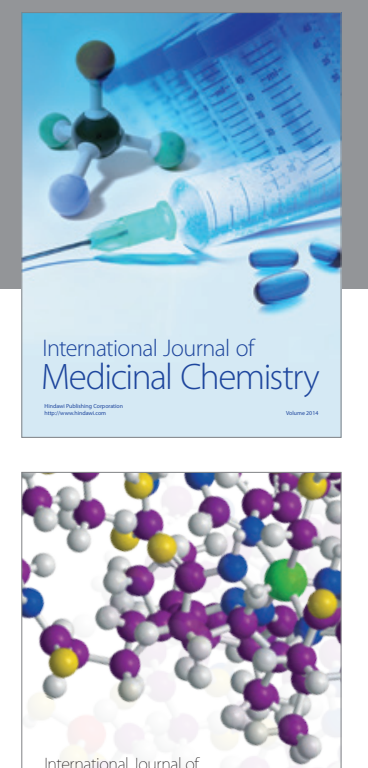

\section{Carbohydrate} Chemistry

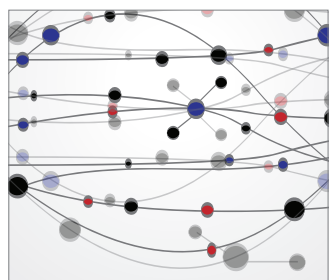

The Scientific World Journal
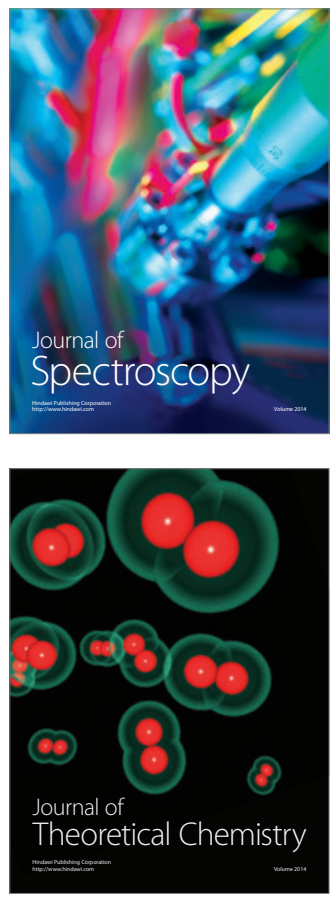
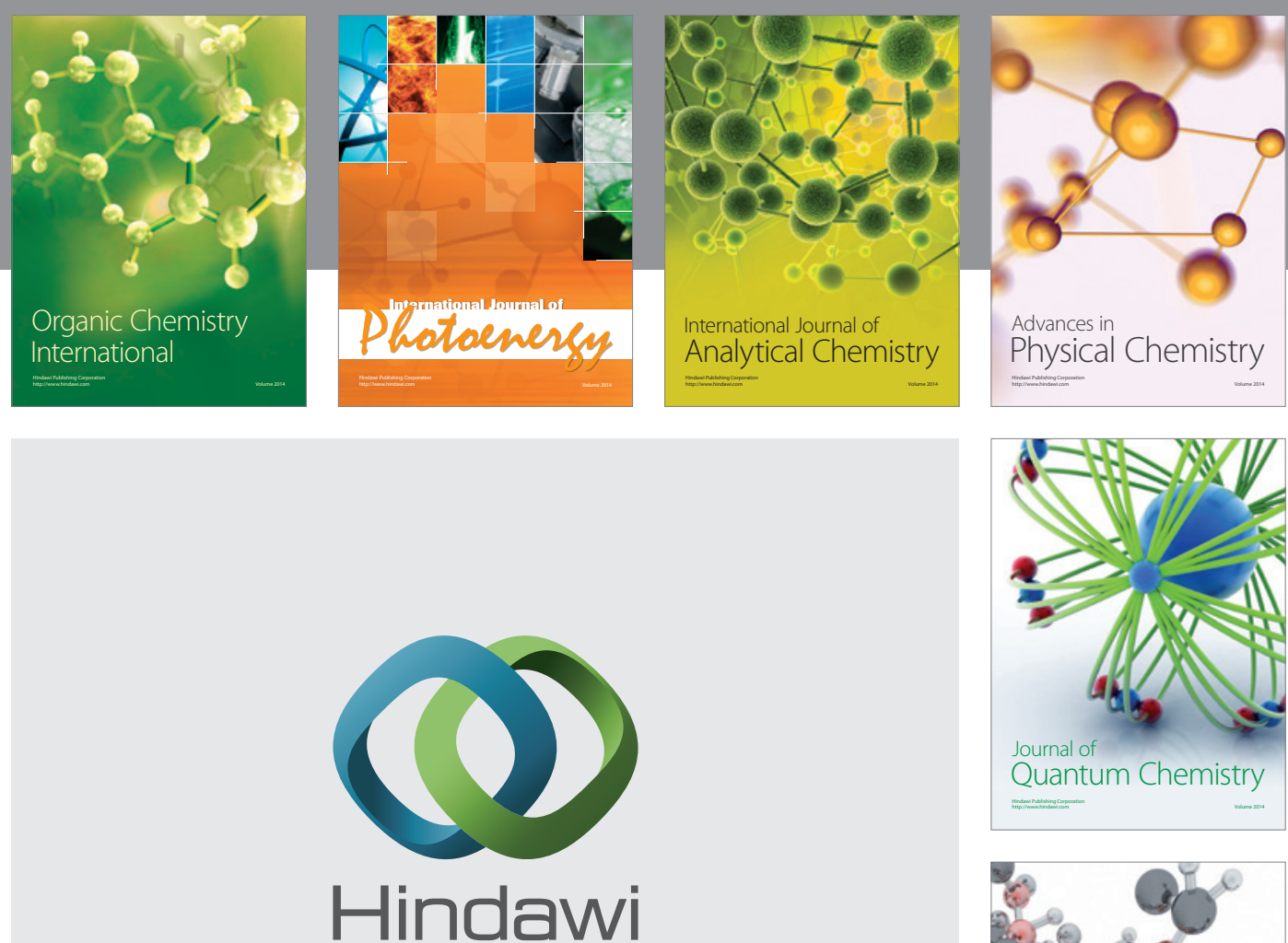

Submit your manuscripts at

http://www.hindawi.com

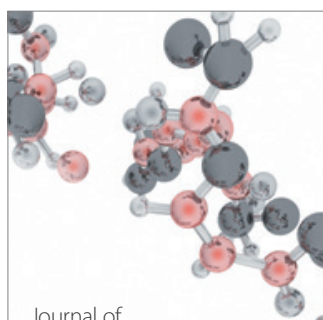

Analytical Methods

in Chemistry

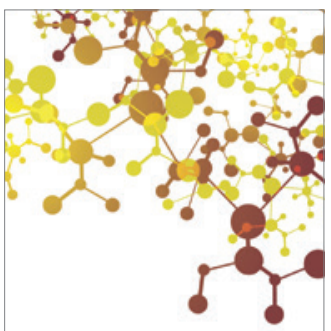

Journal of

Applied Chemistry

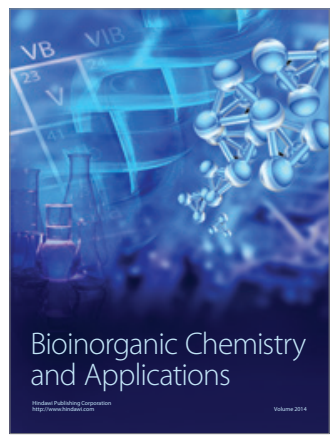

Inorganic Chemistry
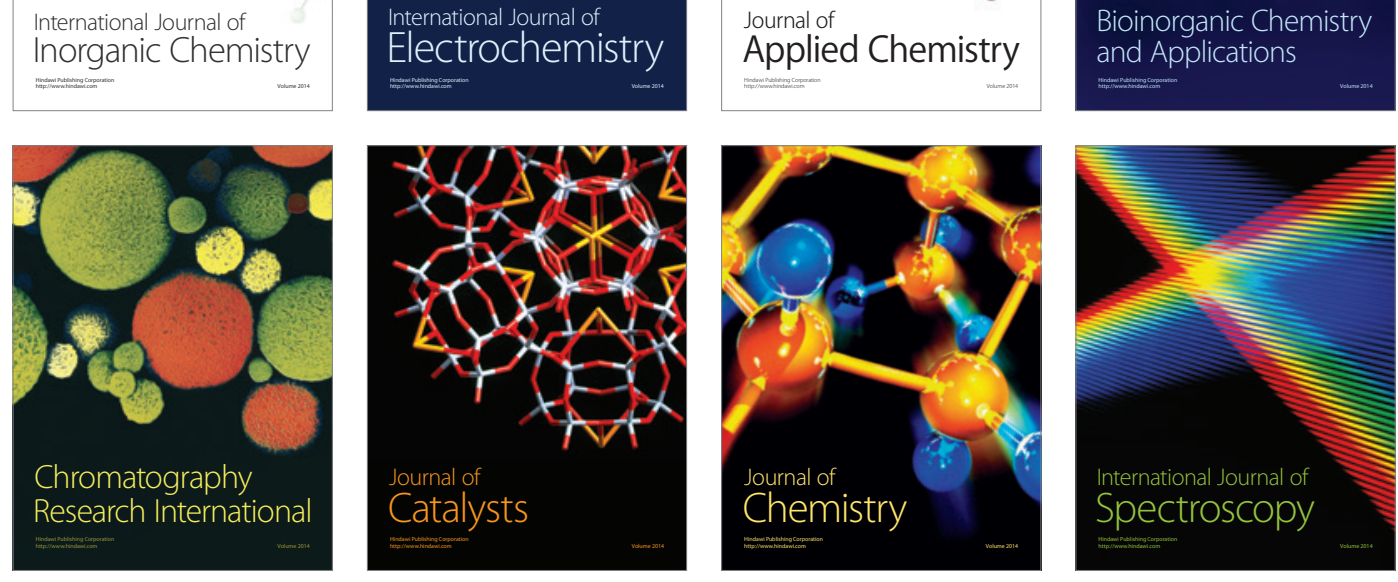